\title{
Fast Method for Computing the Number of Primes Less than a Given Limit
}

\author{
By David C. Mapes
}

Abstract. "Fast Method for Computing the Number of Primes Less Than a Given Limit" describes three processes used during the course of calculation. In the first part of the paper the author proves:

$$
\phi(x, a)=\phi(x, 1)-\phi\left(\frac{x}{p_{2}}, 1\right)-\phi\left(\frac{x}{p_{3}}, 2\right)-\cdots-\phi\left(\frac{x}{p_{a}}, a-1\right)
$$

where $\phi(x, a)$ represents the number of numbers less than or equal to $x$ and not divisible by the first " $a$ " primes. This identity is used to evaluate the formula $\pi(x)=\phi(x, a)+a-1, a+1>\pi(\sqrt{ } x)$ where resulting terms of the form $\phi\left(x^{\prime}, a^{\prime}\right)$ are broken down still further by the previously described method, or numerically evaluated using one or both of two other identities, the choice being dependent on $x^{\prime}$ and $a^{\prime}$.

Following the paper is a table of calculations made using this process which gives the values of $\pi(x)$ for $x$ at intervals of 10 million up to 1000 million, along with the Riemann and the Chebyshev approximations for $\pi(x)$ and the amount they deviate from the true count.

\section{Definitions and Notations.}

$\pi(x)$ : the number of primes less than or equal to $x$.

$p_{a}$ : the $a$ th prime, $\left(p_{1}=2\right)$.

$m_{a}: p_{1} p_{2} \cdots p_{a}$.

$[x]:$ greatest integer $\leqq x$.

$c$ : any integer.

$c \mid x: \quad c$ divides $x$.

$c \nmid x: \quad c$ does not divide $x$.

$S(x, a)$ : set of all numbers $\leqq x$ and prime to $m_{a}$.

$\phi(x, a)$ : number of members of $S(x, a)$.

$\phi(-x, a)$ : equivalent to $-\phi(x, a)$.

$P_{s}(x, a):$ number of products of $s$ primes belonging to $S(x, a), P_{0}(x, a)=1$.

$P_{s}(-x, a):$ equivalent to $-P_{s}(x, a)$.

$P_{1}(x, a)$ : by definition is $\pi(x)-a$.

Legendre Sum: $\phi(x, a)=[x]-\sum_{i \leqq a}\left[x / p_{i}\right]+\sum_{j<i \leqq a}\left[x / p_{i} p_{j}\right]$

$$
-\sum_{k<j<i \leqq a}\left[x / p_{i} p_{j} p_{k}\right]+\cdots
$$

2. Formula Development. Let:

$$
T_{k}(x, a) \equiv(-1)^{\beta_{0}+\beta_{1}+\ldots+\beta_{a-1}}\left[x / p_{1}^{\beta_{0}} p_{2}^{\beta_{1}} \cdots p_{a}^{\beta_{a-1}}\right],
$$

where $k=2^{a-1} \beta_{a-1}+2^{a-2} \beta_{a-2}+\cdots 2^{0} \beta_{0}$ such that the $\beta$ 's are either 1 or 0 , thus

Received June 7, 1962. 
being the digits of $k$ expressed in binary form and where $0 \leqq k<2^{a}$. Let us also adopt: $T_{k}(-x, a) \equiv-T_{k}(x, a)$. Legendre's sum may be written in the following order:

$$
\phi(x, a)=\sum_{k=0}^{2^{a}-1} T_{k}(x, a) .
$$

Let $M$ be an integer $<2^{a}$ and let $2^{i}$ be the highest power of 2 dividing $M$. Let

$$
\gamma(M, x, a)=\sum_{k=M}^{M+2^{i}-1} T_{k}(x, a) .
$$

Full understanding of (3) should be clear from the identity:

(4) $\phi(x, a)=T_{0}(x, a)+\gamma\left(2^{0}, x, a\right)+\gamma\left(2^{1}, x, a\right)+\cdots+\gamma\left(2^{a-1}, x, a\right)$, which is (2) with (3) applied.

From (1) it follows that:

$$
\left\{\operatorname{sgn} T_{k}(x, a)\right\}_{k>0,2^{i} \mid k}=(-1)^{\beta_{i}+\beta_{i+1}+\ldots+\beta_{a-1}}
$$

and

$$
\left|T_{k}(x, a)\right|_{k>0,2^{i} \mid k}=\left[x / p_{i+1}^{\beta_{i}} p_{i+2}^{\beta_{i+1}} \cdots p_{a}^{\beta_{a-1}}\right] .
$$

By substituting $T_{k}(x, a)$ for $x$ and $i$ for $a$ in (1) we get:

$$
\begin{aligned}
T_{k^{\prime}}\left\{T_{k}(x, a), i\right\}=\operatorname{sgn}\left\{T_{k}(x, a)\right\}(-1)^{\beta_{0}{ }^{\prime}+\beta_{1}{ }^{\prime}+\ldots+\beta_{i-1}{ }^{\prime}} & \\
& \times\left[\left|T_{k}(x, a)\right| / p_{1}^{\beta_{0}{ }^{\prime}} p_{2}^{\beta_{1}{ }^{\prime}} \cdots p_{i}^{\beta_{i}{ }^{\prime}}\right],
\end{aligned}
$$

where $k^{\prime}=2^{i-1} \beta_{i-1}^{\prime}+2^{i-2} \beta_{i-2}^{\prime}+\cdots+2^{0} \beta_{0}{ }^{\prime}$ and $0 \leqq k^{\prime}$. When $2^{i} \mid k$, we can substitute (5) and (6) into (7) giving:

$$
T_{k^{\prime}}\left\{T_{k}(x, a), i\right\}_{2^{i} \mid k}=T_{k+k^{\prime}}(x, a) .
$$

By substituting $T_{M}(x, a)$ for $x$ and $i$ for $a$ in (2) we get

$$
\phi\left\{T_{M}(x, a), i\right\}=\sum_{k^{\prime}=0}^{2^{i}-1} T_{k^{\prime}}\left\{T_{M}(x, a), i\right\} .
$$

Using (8), (9) becomes

$$
\phi\left\{T_{M}(x, a), i\right\}_{2^{i} \mid M}=\sum_{k=M}^{M+2^{i}-1} T_{k}(x, a)
$$

and using (3) we have

$$
\gamma(M, x, a)=\phi\left\{T_{M}(x, a), i\right\}
$$

Now (4) becomes

$$
\begin{aligned}
\phi(x, a)=T_{0}(x, a)+\phi\{ & \left.T_{2^{0}}(x, a), 0\right\} \\
& +\phi\left\{T_{2^{i}}(x, a), 1\right\}+\cdots+\phi\left\{T_{2^{a-1}}(x, a), a-1\right\} .
\end{aligned}
$$

By replacing $x$ with $T_{M}(x, a)$ and $a$ with $i$, we get

$$
\begin{aligned}
& \phi\left\{T_{M}(x, a), i\right\}=T_{0}\left\{T_{M}(x, a), i\right\}+\phi\left(T_{2^{0}}\left\{T_{M}(x, a), i\right\}, 0\right) \\
& +\phi\left(T_{2^{1}}\left\{T_{M}(x, a), i\right\}, 1\right)+\cdots+\phi\left(T_{2^{i-1}}\left\{T_{M}(x, a), i\right\}, i-1\right) .
\end{aligned}
$$


Using (1) to find $T_{0}\left\{T_{M}(x, a), i\right\}$ and (8) in general, we get

$$
\begin{aligned}
\phi\left\{T_{M}(x, a), i\right\}_{2^{i} \mid M}= & T_{M}(x, a)+\phi\left\{T_{M+2^{0}}(x, a), 0\right\} \\
& +\phi\left\{T_{M+2^{1}}(x, a), 1\right\}+\cdots+\phi\left\{T_{M+2^{i-1}}(x, a), i-1\right\} .
\end{aligned}
$$

We can now calculate $\phi(x, a)$ by (13) setting $\phi(x, a)$ equal to $\phi\left\{T_{0}(x, a), a\right\}$ where $2^{a} \mid 0$. In (13) $T_{M}(x, a)$ is computed by (1) while the remaining terms are computed by reapplication of (13) or, if reduced by (1) to a numerical expression of $\phi(x, a)$, by any other method of computing $\phi(x, a)$.

3. Other Methods of Calculating $\phi(x, a)$. The following method for calculating $\phi(x, a)$ was developed by D. H. Lehmer [1]. Meissel's formula for finding $\pi(x)$ is a special case of this method [1].

$$
\phi(x, a)=\sum_{s=0}^{v-1} P_{s}(x, a), x<p_{a+1}^{v}
$$

where

$$
\begin{aligned}
& P_{0}(x, a)=1 \\
& P_{1}(x, a)=\pi(x)-a \\
& P_{2}(x, a)=\sum_{p_{a}<p_{i} \leqq x / p_{i}}\left\{\pi\left(x / p_{i}\right)-(i-1)\right\}, \\
& P_{3}(x, a)=\sum_{p_{a}<p_{i} \leqq x / p_{i}{ }^{2}} \sum_{p_{i} \leqq p_{j} \leqq x / p_{i} p_{j}}\left\{\pi\left(x / p_{i} p_{j}\right)-(j-1)\right\} .
\end{aligned}
$$

If $V-1$ is greater than 3 this method of calculating $\phi(x, a)$ may not be practical. Also

$$
\phi(x, a)=c \phi\left(m_{a}, a\right)+\phi(r, a)
$$

where $x=c m_{a}+r,|r| \leqq \frac{1}{2} m_{a}$, and $a>1$. Hence $c \phi\left(m_{a}, a\right)$ can be calculated using a table of the values for $\phi\left(m_{a}, a\right)$ and $\phi(r, a)$ by (14) or (13).

4. Fast Method for Computing $\boldsymbol{\pi}(\mathbf{x})$. From the above development we can formulate the following procedure for computing $\pi(x)$. Use the formula:

$$
\pi(x)=\phi(x, a)+a-1
$$

where $p_{a+1}^{2} \geqq x \geqq{p_{a}}^{2}$. Place a limit on $V$ in (14) such that $\phi(x, a)$ is not computable by (14) when $p_{a+1}^{4} \leqq x$. Now place a limit on $a$ in (15) such that $\phi(r, a)$ will always be computable by (14) with its restriction $V \leqq 4$. This means the value of $a$ in (15) must meet the requirement $1<a \leqq 6$. With a limited table of primes, the larger the better, we are prepared to compute $\pi(x)$.

Compute $\phi(x, a)$ in (16) by (13) where the resulting term $T_{0}(x, a)$ is computed by (1). The rest of the resulting terms from (13) considered as expressions of the form $\phi(x, a)$ are computed by (1) if $a=1$, by $(15), \phi(r, a)$ being computed by (14), if $1<a \leqq 6$, and by (14) or the reapplication of (13) if $a>6$. In the latter case $\phi(x, a)$ is computed by (14) unless $V>4$ or $\pi(x)$ is too large to be found in the table of primes when $2 \leqq V \leqq 4$. The resulting terms from a reapplication of (13) are calculated using the process used in calculating the resulting terms of the calculation of $\phi(x, a)$ in $(16)$. 


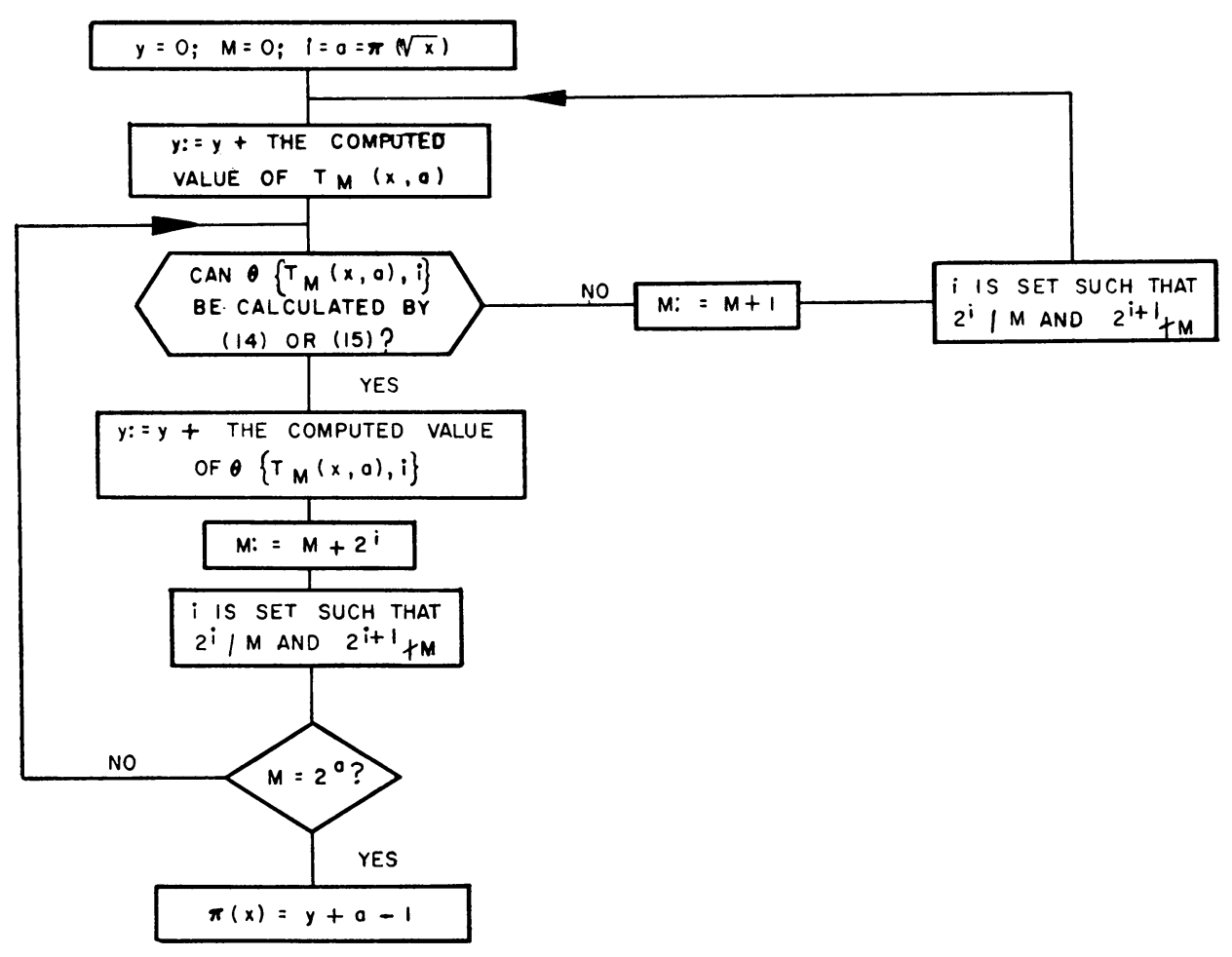

We now introduce a system of order as an aid to our method of calculating $\pi(x)$. At any point in our calculations we will have a number of terms calculated. Introducing $y$ as the sum of these calculated terms, here is the system of order in flow chart form.

The length of time necessary for the calculation of $\pi(x)$ on the 709 using the author's program was:

$$
t \sim 60 x^{\log _{10} 5} \mu \text { sec. }
$$

In the following table the " $L i$ " function is Chebyshev's approximation for $\pi(x)$ and the " $R$ " function is the Riemann approximation

$$
\begin{aligned}
L i(x) & =\int_{2}^{x} \frac{d t}{\ln t}, \\
R(x) & =\sum_{n=1}^{\infty} n^{-1} \mu(n) \operatorname{Li}\left(x^{1 / n}\right) .
\end{aligned}
$$

They are computed using

$$
\begin{gathered}
L i(x)=\gamma+\ln (\ln x)+\ln x+\frac{(\ln x)^{2}}{2 \times 2 !}+\frac{(\ln x)^{3}}{3 \times 3 !}+\cdots+\frac{(\ln x)^{75}}{75 \times 75 !}, \\
R(x)=1+\frac{\ln x}{S_{2}}+\frac{(\ln x)^{2}}{S_{3}(2 \times 2 !)}+\cdots+\frac{(\ln x)^{75}}{S_{76}(75 \times 75 !)}
\end{gathered}
$$

where $S_{n}=\sum_{1}^{\infty} k^{-n}$. 
Although the table gives $x$ only at intervals of 10 million, calculations have also been made for $x$ at intervals of 1 million, with the results listed in the same manner. Values of $\pi(x)$ for $x$ (in millions) at $1,2, \ldots, 10$ checked with values obtained by D. N. Lehmer [2]. The values of $\pi(x)$ for the following values of $x$ (in millions) checked with those listed by D. H. Lehmer [1, p. 386]: 20, 25, 33, 37, 40, 90, 100, 999 , and 1000 .

5. Acknowledgement. Work was performed under the auspices of the U. S. Atomic Energy Commission. Formula construction was done with the aid of D. H. Lehmer. Tables were computed using an IBM 709 at the Lawrence Radiation Laboratory of the University of California.

Lawrence Radiation Laboratory

University of California

Livermore, California

1. D. H. LeHMeR, Illinois J. Math., v. 3, no. 3, p. 381-388, 1959. 1956 .

2. D. N. Lehmer, List of Prime Numbers from 1 to 10,006,721, New York, Hafner Pub. Co., 
Comparison of the Count of Primes with the Corresponding Values of the Formulas of Chebyshev and Riemann at intervals of 10,000,000 up to 1,000,000,000.

\begin{tabular}{|c|c|c|c|c|c|}
\hline$x$ & $\pi(x)$ & $\operatorname{Li}(x)$ & $R(x)$ & $\begin{array}{c}L i(x)- \\
\quad \pi(x)\end{array}$ & $\begin{array}{c}R(x)- \\
\pi(x)\end{array}$ \\
\hline 10000000 & 664579 & 664918 & 664667 & +339 & +88 \\
\hline 20000000 & 1270607 & 1270905 & 1270571 & +298 & -36 \\
\hline 30000000 & 1857859 & 1858213 & 1857818 & +354 & -41 \\
\hline 40000000 & 2433654 & 2434016 & 2433570 & +362 & -84 \\
\hline 50000000 & 3001134 & 3001557 & 3001067 & +423 & -67 \\
\hline 60000000 & 3562115 & 3562683 & 3562154 & $\begin{array}{r}+568 \\
\end{array}$ & +39 \\
\hline 70000000 & 4118064 & 4118585 & 4118020 & +521 & -44 \\
\hline 80000000 & 4669382 & 4670091 & 4669493 & +709 & +111 \\
\hline 90000000 & 5216954 & 5217810 & 5217182 & +856 & +228 \\
\hline 100000000 & 5761455 & 5762209 & 5761552 & +754 & $\begin{array}{r}+97 \\
\end{array}$ \\
\hline 110000000 & 6303309 & 6303656 & 6302971 & +347 & -338 \\
\hline 120000000 & 6841648 & 6842446 & 6841735 & +798 & +87 \\
\hline 130000000 & 7378187 & 7378825 & 7378090 & +638 & -97 \\
\hline 140000000 & 7912199 & 7912998 & 7912239 & $\begin{array}{r}+799 \\
+\end{array}$ & +40 \\
\hline 150000000 & 8444396 & 8445139 & 8444357 & +743 & -39 \\
\hline 160000000 & 8974458 & 8975397 & 8974593 & +939 & +135 \\
\hline 170000000 & 9503083 & 9503902 & 9503077 & +819 & -6 \\
\hline 180000000 & 10030385 & 10030768 & 10029923 & +383 & -462 \\
\hline 190000000 & 10555473 & 10556096 & 10555231 & +623 & -242 \\
\hline 200000000 & 11078937 & 11079975 & 11079090 & +1038 & +153 \\
\hline 210000000 & 11601626 & 11602484 & 11601581 & +858 & $-4 \tilde{5}$ \\
\hline 220000000 & 12122540 & 12123696 & 12122775 & +1156 & +235 \\
\hline 230000000 & 12642573 & 12643676 & 12642736 & +1103 & +163 \\
\hline 240000000 & 13161544 & 13162482 & 13161525 & $\begin{array}{r}+938 \\
\end{array}$ & -19 \\
\hline 250000000 & 13679318 & 13680169 & 13679195 & +851 & -123 \\
\hline 260000000 & 14195860 & 14196786 & 14195796 & +926 & -64 \\
\hline 270000000 & 14711384 & 14712378 & 14711372 & $\begin{array}{r}+994 \\
\end{array}$ & -12 \\
\hline 280000000 & 15226069 & 15) 226988 & 15225965 & +919 & -104 \\
\hline 290000000 & 15739663 & 15740653 & 15739614 & +990 & -49 \\
\hline 300000000 & 16252325 & 16253409 & 16252355 & +1084 & +30 \\
\hline 310000000 & 16764521 & 16765291 & 16764222 & +770 & -299 \\
\hline 320000000 & 17275206 & 17276328 & 17275245 & +1122 & +39 \\
\hline 330000000 & 17785475 & 17786551 & 17785453 & +1076 & -22 \\
\hline 340000000 & 18294605 & 18295985 & 18294873 & +1380 & +268 \\
\hline 350000000 & 18803526 & 18804658 & 18803531 & +1132 & +5 \\
\hline 360000000 & 19311288 & 19312592 & 19311452 & +1304 & +164 \\
\hline 370000000 & 19818405 & 19819810 & 19818656 & +1405 & +251 \\
\hline 380000000 & 20325373 & 20326334 & 20325167 & $\begin{array}{r}+961 \\
\end{array}$ & -206 \\
\hline 390000000 & 20831210 & 20832184 & 20831003 & +974 & -207 \\
\hline 400000000 & 21336326 & 21337378 & $2133618 \overline{5}$ & +1052 & -141 \\
\hline 410000000 & 21840713 & 21841935 & 21840729 & +1222 & +16 \\
\hline 420000000 & 22344479 & 22345872 & 22344653 & +1393 & +174 \\
\hline 430000000 & 22848050 & 22849204 & 22847973 & +1154 & -77 \\
\hline 440000000 & 23350555 & 23351948 & 23350705 & +1393 & +150 \\
\hline 450000000 & 23853038 & 23854119 & 23852863 & +1081 & -175 \\
\hline 460000000 & 24354548 & 24355729 & 24354461 & +1181 & -87 \\
\hline 470000000 & 24855718 & 24856793 & 24855513 & +1075 & -205 \\
\hline 480000000 & 25356424 & 25357324 & 25356032 & $\begin{array}{r}+900 \\
+\end{array}$ & -392 \\
\hline 490000000 & 25856368 & 25857333 & 25856029 & +965 & -339 \\
\hline 500000000 & 26355867 & 26356832 & 26355517 & +965 & -350 \\
\hline
\end{tabular}




\begin{tabular}{|c|c|c|c|c|c|}
\hline$x$ & $\pi(x)$ & $\operatorname{Li}(x)$ & $R(x)$ & $\begin{array}{c}\operatorname{Li}(x)- \\
\pi(x)\end{array}$ & $\begin{array}{c}R(x)- \\
\pi(x)\end{array}$ \\
\hline$j 10000000$ & 26854252 & 26855833 & $268 \tilde{5} 4507$ & +1581 & $+25 \tilde{5}$ \\
\hline 520000000 & $273 \tilde{5} 2687$ & 27354346 & 27353008 & $+16 \tilde{9} 9$ & +321 \\
\hline 530000000 & 27850698 & 27852381 & 27851033 & +1683 & +335 \\
\hline 540000000 & 28348381 & 28349949 & 28348589 & +1568 & +208 \\
\hline ;);0000000 & 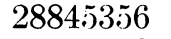 & 28847059 & 28845688 & +1703 & +332 \\
\hline$j 60000000$ & 29342150 & 29343720 & 29342338 & +1570 & +188 \\
\hline 570000000 & 29838286 & 29839940 & 29838548 & +1654 & +262 \\
\hline 580000000 & 30334175 & 30335730 & 30334327 & +1555 & +152 \\
\hline 590000000 & 30829544 & 30831095 & 30829682 & +1551 & +138 \\
\hline 600000000 & 31324703 & 31326045 & 31324622 & +1342 & -81 \\
\hline 610000000 & 31819444 & 31820587 & 31819153 & +1143 & -291 \\
\hline 620000000 & 32313388 & 32314729 & 32313285 & +1341 & -103 \\
\hline 630000000 & 32807229 & 32808477 & 32807023 & +1248 & -206 \\
\hline 640000000 & 33300450 & 33301838 & 33300374 & +1388 & -76 \\
\hline 650000000 & 33793395 & 33794819 & 33793345 & +1424 & -50 \\
\hline 660000000 & 34286170 & 34287427 & 34285943 & +1257 & -227 \\
\hline 670000000 & 34778319 & 34779667 & 34778173 & +1348 & -146 \\
\hline 680000000 & 35270167 & 35271546 & 35270042 & +1379 & -125 \\
\hline 690000000 & 35761747 & 35763069 & 35761556 & +1322 & -191 \\
\hline 700000000 & 36252931 & 36254242 & 36252719 & +1311 & -212 \\
\hline 710000000 & 36743905 & 36745071 & 36743539 & +1166 & -366 \\
\hline 720000000 & 37234048 & 37235561 & 37234019 & +1513 & -29 \\
\hline 730000000 & 37724170 & 37725717 & 37724166 & +1547 & -4 \\
\hline 740000000 & 38213987 & 38215544 & 38213984 & +1557 & -3 \\
\hline 750000000 & 38703181 & 38705046 & 38703477 & +1865 & +296 \\
\hline 760000000 & 39192219 & 39194230 & 39192652 & +2011 & +433 \\
\hline 770000000 & 39680979 & 39683099 & 39681512 & +2120 & +533 \\
\hline 780000000 & 40169476 & 40171658 & 40170062 & +2182 & +586 \\
\hline 790000000 & 40658253 & 40659911 & 40658306 & +1658 & +53 \\
\hline 800000000 & 41146179 & 41147862 & 41146248 & +1683 & +69 \\
\hline 810000000 & 41634187 & 41635516 & 41633893 & +1329 & -294 \\
\hline 820000000 & 42121502 & 42122877 & 42121245 & +1375 & -257 \\
\hline 830000000 & 42608404 & 42609948 & 42608308 & +1544 & -96 \\
\hline 840000000 & 43095410 & 43096733 & 43095084 & +1323 & -326 \\
\hline 850000000 & 43581966 & 43583236 & 43581579 & +1270 & -387 \\
\hline 860000000 & 44067840 & 44069462 & 44067796 & +1622 & -44 \\
\hline 870000000 & 44553888 & 44555412 & 44553738 & +1524 & $-1 \tilde{5} 0$ \\
\hline 880000000 & 45039361 & 45041091 & 45039408 & +1730 & +47 \\
\hline 890000000 & 45524412 & 45526502 & 45524811 & +2090 & +399 \\
\hline 900000000 & 46009215 & 46011649 & 46009949 & +2434 & +734 \\
\hline 910000000 & 46494557 & 46496534 & 46494826 & +1977 & +269 \\
\hline 920000000 & 46979583 & 46981161 & 46979445 & +1578 & -138 \\
\hline 930000000 & 47463433 & 47465532 & 47463809 & +2099 & +376 \\
\hline 940000000 & 47947424 & 47949652 & 47947920 & +2228 & +496 \\
\hline 950000000 & 48431471 & 48433523 & 48431783 & +2052 & +312 \\
\hline 960000000 & 48915316 & 48917147 & 48915399 & +1831 & +83 \\
\hline 970000000 & 49398798 & 49400527 & 49398771 & +1729 & -27 \\
\hline 980000000 & 49881580 & 49883667 & 49881903 & +2087 & +323 \\
\hline 990000000 & 50364709 & 50366569 & 50364797 & +1860 & +88 \\
\hline 1000000000 & 50847534 & 50849235 & 50847455 & +1701 & -79 \\
\hline
\end{tabular}

